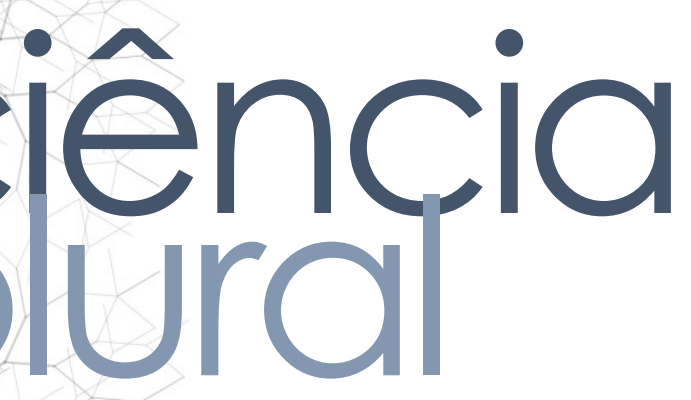

\title{
GRAVIDEZ NA ADOLESCÊNCIA: UMA ANÁLISE DO PERFIL DAS ADOLESCENTES ASSISTIDAS EM HOSPITAL ESCOLA NA CIDADE DE MACEIÓ-AL
}

Pregnancy in adolescence: An analysis of the profile of adolescents assisted in a school hospital in the city of Maceió-AL

Embarazo em adolescencia: Um análisis del perfil de adolescentes assistidas em um hospital escolar de la ciudad de Maceió-AL

Renata Vasconcelos de Carvalho - Aluna do Curso de Medicina - Centro Universitário Tiradentes Alagoas-UNIT- AL• E-mail: renatacarvalhov5@gmail.com

Isis Carvalho Miranda - Aluna do Curso de Medicina - Centro Universitário Tiradentes Alagoas-UNIT- AL・E-mail: mirandacisis@hotmail.com

Antônio Carlos da Rocha Moraes - Professor • Centro Universitário Tiradentes Alagoas-UNIT- AL・E-mail: acrmoraes@uol.com.br

Ronaldo Gomes Alvim • Professor • Centro Universitário Tiradentes Alagoas-UNITAL・E-mail: alvimrg@yahoo.com.br

Autora correspondente:

Renata Vasconcelos de Carvalho • E-mail: renatacarvalhov5@gmail.com 


\section{RESUMO}

Introdução: A adolescência é um período curto, porém é nela que ocorrem mudanças biológicas, físicas e psicológicas determinantes na busca da autoafirmação e que podem acarretar complicações no ciclo de vida. A gravidez vista nesta etapa é uma realidade no Brasil e, apesar de susceptível em qualquer classe social, os fatores econômico e social são determinantes nos índices estatísticos. Objetivo: Analisar o perfil das adolescentes que utilizam os serviços do ambulatório e enfermaria de obstetrícia do Hospital Veredas, identificar os fatores que levaram a uma maternidade precoce, as relações sociais, familiares e escolares das adolescentes, além das suas expectativas futuras na construção afetiva da relação mãe-filho e dos possíveis riscos gerados à saúde de ambos. Metodologia: Pesquisa analítica, individual, transversal e observacional, com dados coletados através de análise de prontuários para seleção da amostra e entrevistas com as gestantes do ambulatório e enfermaria de obstetrícia do Hospital Veredas, Maceió-Alagoas, entre janeiro de 2020 e novembro de 2020. Resultados e Discussão: Das adolescentes analisadas, 52,3\% eram menores de idade, dado significativo que demonstra a natureza social destas gestações. Quando questionadas sobre sua percepção quanto a gestação, 57,5\% declararam que se tratava de uma gravidez indesejada, informação que associada a narrativa dessas mulheres, demonstra que a gravidez associada a ausência de uma rede de apoio, agrava sua rejeição, refletindo em um isolamento e dificuldade de aceitação. Pode-se constatar através de análise inferencial que uma tendência das adolescentes etilistas evoluírem com gestações pré-termo (tempo de gestação < 37 semanas) e recém-nascidos prematuros. Conclusões: Através da análise dos dados e da bibliografia analisada, nota-se que a gravidez na adolescência é uma realidade no município de Maceió, e permanece um importante problema de saúde pública gerando impactos negativos nos aspectos sociais, econômicos e nos indicadores de saúde dessas jovens.

Palavras-Chave: Família; Gravidez na adolescência; Sexualidade.

\section{ABSTRACT}

Introduction: Adolescence is a short period, but it is when biological, physical and psycological changes happen and those are decisive in the search for self-affirmation and can cause complications in the life cycle. Teenage pregnancy is a reality in Brazil and, although susceptible in any social class, the economic and social factors are determinant in the statistical indexes. Objective: To analyze the profile of adolescents who use the services of the ambulatory and obstetric ward of Hospital Veredas, identifying the factors that led to an early motherhood, the social, family and school relationships of the adolescents, in addition to their future expectations in the affective construction the mother-child relationship and the possible risks to their health. ethodology: Analytical, individual, cross-sectional and observational research, with ata collected through analysis of medical records for sample selection and interviews ith pregnant women from the outpatient clinic and obstetrics ward of Hospital redas, Maceió-Alagoas, between January 2020 to November 2020. Results and cussion: Of the adolescents analyzed, 52.3\% were minors, a significant figure that 
reflects the social nature of these pregnancies. When asked about their perception of pregnancy, $57.5 \%$ stated that it was an unwanted pregnancy, given that associated with the narrative of these women, demonstrates that pregnancy associated with the absence of a support network, aggravates their rejection, reflecting on a isolation and difficulty of acceptance. It can be seen through inferential analysis that a tendency for alcooholic teenagers to evolve with preterm pregnancies (gestation time $<37$ weeks) and premature newborns. Conclusions: Through the analysis of the data and the analyzed bibliography, it is noted that teenage pregnancy is a reality in the city of Maceió and remains an important public health, problem generating negative impacts on the social, economic and health indicators of these young women.

Keywords: Family; Teenage pregnancy; Sexuality.

\section{RESUMEN}

Introducción: La adolescencia es un período corto, donde ocurren los cambios biológicos, físicos y psicológicos que son determinantes en la búsqueda de la autoafirmación y que pueden derivar en complicaciones en el ciclo vital. El embarazo visto en esta etapa es una realidad en Brasil y, aunque susceptible en cualquier clase social, los factores económicos y sociales son determinantes. Objetivo: Analizar el perfil de las adolescentes que utilizan los servicios del ambulatorio y obstétrico del Hospital Veredas, para identificar los factores que llevaron a una maternidad temprana, las relaciones sociales, familiares y escolares de las adolescentes, además de sus expectativas futuras en la construcción afectiva. Metodología: Investigación analítica, individual, transversal y observacional, con datos recolectados mediante análisis de historias clínicas para selección de muestras y entrevistas a gestantes del ambulatorio y sala de ginecología y obstetricia del Hospital Veredas, Maceió-Alagoas, entre el Enero de 2020 al Noviembre 2020. Resultados y Discussión: De las analizadas, el 52,3\% fueron menores de edad, cifra significativa que refleja el carácter social de estos embarazos. Sobre su percepción del embarazo, el 57,5\% afirmó que se trató de un embarazo no deseado, información que, asociada a la narrativa de estas mujeres, demuestra que el embarazo asociado a la ausencia de una red de apoyo, agrava su rechazo, reflejándose en una aislamiento y dificultad de aceptación. Se puede ver a través del análisis inferencial que una tendencia de los adolescentes bebedores a evolucionar con los embarazos pretérmino (tiempo de gestación $<37$ semanas) y los recién nacidos prematuros. Conclusiones: A través del análisis de los datos y la bibliografía analizada, se constata que el embarazo adolescente es una realidad en la ciudad de Maceió, y sigue siendo un importante problema de salud pública generando impactos negativos en los indicadores sociales, económicos y de salud de estos jôvenes.

labras clave: Familia; Embarazo en la adolescencia; Sexualidad. 


\section{Introdução}

Segundo a Organização Mundial de Saúde, a adolescência consiste na faixa etária que vai dos 10 aos 19 anos e, apesar de ser considerada um período curto, é nesta fase que ocorrem mudanças de cunho biológico, físico, psicológico e comportamental, processos estes, determinantes na busca da autoafirmação e que tendem a acarretar complicações no ciclo de vida do indivíduo no futuro ${ }^{1}$.

Quando se percorre pelos registros históricos da mulher brasileira e toda sua trajetória de lutas para se autoafirmar na sociedade, nos deparamos com o fato de que apesar dos avanços alcançados, quanto à liberdade e direitos das mulheres, que se relacionam diretamente a maior formação educacional feminina e ao aumento importante da sua atuação no mercado de trabalho, o valor sociocultural da mulher ainda perpassa por argumentos religiosos e sociais, e com todas as suas pluralidades, permanece atrelado a um papel primordial, a maternidade ${ }^{2}$.

Devido ao processo de extinção da cultura do casamento arranjado no ocidente e redução da submissão da mulher no ambiente caseiro, pode-se comprovar que a gestação na adolescência não é mais uma imposição da família e sim uma inversão dos valores econômicos, culturais e sociais devido ao contexto no qual estas jovens estão inseridas ${ }^{3}$.

Em um país tão iníquo como o Brasil, existe uma associação quanto a falta de uma perspectiva de vida alternativa à maternidade, decorrente da ausência de uma educação de qualidade e da possibilidade de inserção no mercado de trabalho formal, trazendo a visão da família como projeto de vida, acrescido a falta de acesso à informação reprodutiva de qualidade no currículo escolar ${ }^{2}$. Desta forma, a gravidez na adolescência, ocorre em sua maioria de forma não intencional, e traz consigo diversos fatores desfavoráveis em relação ao ambiente de convívio dessas mulheres ${ }^{4}$.

Nesta perspectiva, acresce ao fato de que ser mãe nesta etapa da vida resulta diversas implicações como: abandono das atividades escolares, riscos para o feto e ra a mãe, conflitos familiares, discriminação social, afastamento de grupos de vivência, adiamento ou destruição de sonhos e planos, associados aos sentimentos 
de perda, tristeza, solidão, isolamento e preocupações que podem surgir em consequência da gestação na adolescência 5 .

No Brasil, a taxa de gravidez na adolescência é alta, atingindo o valor de 400 mil casos/ano, número bastante superior quando comparado a países desenvolvidos. No ano de 2015, 18\% dos brasileiros nascido vivos eram fruto de uma gravidez na adolescência, destacando-se estados da região Nordeste, concentrando 180 mil casos, ou seja, $32 \%$ do total avaliado ${ }^{6}$. Dados como estes refletem que o caráter social não só permeia a realidade destas adolescentes como chega a ser determinante nas estatísticas nacionais.

De acordo com dados da OMS, a mortalidade materna está entre as principais causas de óbito entre 15-24 anos na América, e quando ocorre na faixa de mulheres inferiores a 15 anos, pode até mesmo duplicar o risco de morte materna ${ }^{7}$. Alguns fatores estão diretamente relacionados com um maior risco dessas gestações, dentre eles: Idade menor que 16 anos ou menarca há menos de dois anos (fenômeno de duplo anabolismo: competição biológica entre mãe e feto pelos mesmos nutrientes, pois a gestante ainda encontra-se em desenvolvimento final); altura inferior a $150 \mathrm{~cm}$ ou peso menor que 45kg; uso de álcool, cigarro ou outras drogas ilícitas; gestações decorrentes de violência sexual; presença de atitudes negativas quanto à gestação ou rejeição ao feto; tentativas de interromper a gestação; existência de dificuldades de acesso e acompanhamento aos serviços de pré-natal; não realização do pré-natal ou pré-natal incompleto; presença de doenças crônicas; presenças de doenças agudas e emergentes (dengue, zika, toxoplasmose, dentre outras); ocorrência de pré-eclâmpsia ou desproporção pélvica-fetal, gravidez gemelar e complicações obstétricas durante o parto; Falta de conexão ou apoio familiar à adolescente, principalmente de sua própria mãe ou do pai da criança ${ }^{6}$.

De acordo com dados do Sistema de Informações sobre Nascidos Vivos (SINASC), dos 52.496 nascidos vivos no ano de 2018 em Maceió-AL, 22,7\% das uérperas tinham até 19 anos, dado bastante significativo que reflete que a gravidez a adolescência é uma realidade na capital alagoana ${ }^{8}$. 


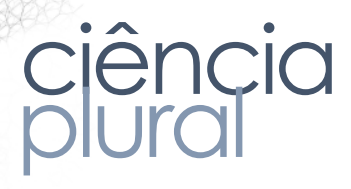

Além disso, durante a revisão de literatura, pode-se perceber que a perspectiva que se apresenta é extremamente relevante, no sentido de que pouca bibliografia recente foi encontrada no que diz respeito ao tema abordado no estado de Alagoas, sendo sua maioria antiga com mais de dez anos de publicação, fazendo-se necessário um estudo mais pormenorizado da situação do estado sobre as circunstâncias em que estão envolvidas a vida gestacional dessas adolescentes, para servir de suporte no desenvolvimento de políticas públicas e consequentemente no controle e prevenção da gravidez prematura no município de Maceió.

Desta forma, buscou-se analisar o perfil das adolescentes que utilizam os serviços de atenção e acolhimento do ambulatório e enfermaria de obstetrícia do Hospital Veredas, objetivando identificar os fatores que levaram o grupo social em questão a uma maternidade precoce, as relações sociais, familiares e escolares das adolescentes durante a gestação, além das suas expectativas futuras na construção afetiva da relação mãe-filho e dos possíveis riscos gerados à saúde de ambos durante a gestação.

\section{Metodologia}

Trata-se de uma pesquisa analítica, individual, transversal e observacional, na qual os dados foram coletados através de entrevistas com as gestantes do ambulatório e enfermaria de obstetrícia do Hospital Veredas, localizado na cidade de Maceió, Alagoas, entre os meses de janeiro de 2020 a novembro de 2020.

Para o desenvolvimento da pesquisa foi realizado estudo prévio, com levantamento de bibliografias relevantes para o tema, nas principais bases de dados PubMed®, SciELO®, LILACS® e Biblioteca Virtual do Ministério da Saúde norteando os questionamentos aplicados e gerando embasamento teórico para o seu desenvolvimento.

Otrabalho de campo contou com duas fases:

A primeira constou de análise de 40 prontuários e atendimentos de adolescentes fase gestacional e puérperas com idades que variavam entre 13 e 19 anos, assistidas 
pela equipe de saúde do hospital, na qual se encaixavam para participação da entrevista baseada nos critérios de inclusão e exclusão do trabalho. Sendo os seguintes critérios de inclusão: realizar pré-natal no ambulatório de obstetrícia do Hospital Veredas ou ter realizado parto no mesmo; declarar interesse em participar da pesquisa; a gravidez não poderá ser resultante de um abuso sexual. Os critérios de exclusão consistem em ter idade igual ou superior a 20 anos, ter procurado o ambulatório de obstetrícia por outras causas que não gravidez e ter gestação decorrente de abuso sexual.

A pesquisa foi baseada segundo as diretrizes e normas regulamentadoras para pesquisa que envolve seres humanos, aprovadas pelo Conselho Nacional de Saúde do Ministério da Saúde, resolução $n^{0} 466 / 12$, tendo como recomendações: esclarecimentos necessários a todos os integrantes, participação voluntária, assinatura do termo de consentimento livre e esclarecido e termo de assentimento livre e esclarecido para aquelas menores de idade, sigilo e guarda das informações, entre outras. Para a realização da segunda fase, foi aprovado no Comitê de Ética em Pesquisa do Centro Universitário Tiradentes (UNIT), com o parecer número 3.814.742.

A segunda parte da pesquisa consistiu na realização de entrevistas privadas com as gestantes acompanhadas ou não pelos seus representantes legais, por meio de um encontro único, numa sala previamente estabelecida cedida pelo Hospital, ou no próprio quarto de enfermaria no qual estava internada a puérpera.

Uma vez selecionada as gestantes, foi-lhes dada uma explicação em relação ao projeto, e posteriormente solicitado autorização para a entrevista após a assinatura do Termo do Consentimento Livre e Esclarecido e do Termo de Assentimento Livre e Esclarecido para as gestantes menores de 18 anos.

As entrevistas foram pensadas no sentido de promover às gestantes um ambiente acolhedor e seguro, proporcionando a captação da individualidade de cada ma delas. Para nortear o encontro as perguntas foram baseadas em um questionário berto, com roteiro pré-estabelecido pelos pesquisadores, no sentido de garantir erdade nas respostas. Durante o diálogo entre pesquisadoras e informantes, foi citado a gravação para que posteriormente as falas fossem transcritas com o intuito 
de fazer uma avaliação mais criteriosa dos encontros para realização das análises. Para cada gestante entrevistada, foi dado um número, mantendo assim o sigilo da sua identidade, através da criação de um código de registro.

Para estudo dos dados, inicialmente foi feita uma análise descritiva, delimitando o perfil das participantes: média da idade, procedência, paridade, tempo de gestação, hábitos de vida (tabagismo e etilismo), intercorrências, desejo de gestar, relacionamento com o parceiro, estado emocional, apoio familiar, relação com o estudo/escola, ciclos de amizade e as expectativas para o futuro afetivo com o filho. Para melhor compreensão desses dados, foi realizado uma pré-análise através de uma leitura inicial e intuitiva, formulando pressupostos e identificando elementos específicos que interferiram no processo.

Após esta etapa, foram utilizados métodos de estatística inferencial por meio da técnica Spearman para correlação de associação das variáveis estudadas e teste de Mann-Whitney para comparação de grupos. Após realização do teste para comparação de grupos, foi utilizada a técnica de Shapiro-Wilk para avaliar a normalidade dos dados numéricos. Os conjuntos de dados não passaram pelo teste de normalidade foram interpretados pelo uso de técnicas não-paramétricas para análise de dados.

Por fim, foi aplicado um estudo analítico através do teste de associação de variáveis quantitativas. Buscando associações entre as variáveis, considerando: paridade; tabagismo; etilismo; gestação desejada ou não desejada; relação com o pai da criança ou não; presença ou ausência de problemas de saúde durante a gestação; estado emocional durante a gravidez (tranquilo ou abalado); se teve apoio familiar ou não; se continuou estudando depois da gravidez; se pretende voltar a estudar depois do parto; se teve apoio dos amigos da escola; e a percepção - positiva ou negativa quanto ao futuro afetivo com o filho. Ao total, foram efetuadas 66 análises.

\section{esultados e Discussão}

\section{perfil das adolescentes grávidas}

Foram analisadas 40 adolescentes entre 13 e 19 anos, estas possuíam uma média 16,9 anos com desvio padrão de 1,7 anos para mais ou para menos, delineando o 


\section{ciência plural}

perfil demonstrado no gráfico (Gráfico 1). Na faixa etária analisada, 52,5\% das adolescentes eram menores de idade, representando a maioria das mulheres, um dado significativo quando se parte para análise da natureza social destas gestações.

Gráfico 1: Idade das adolescentes grávidas entrevistadas. Maceió-AL, 2020.

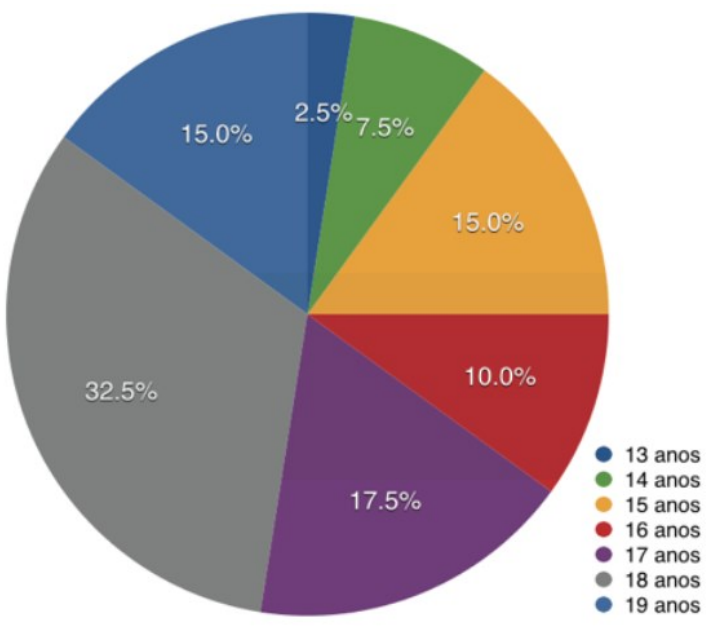

Com relação a procedência, 72,5\% destas mulheres residiam no município de Maceió, das outras 27,5\%, 22,5\% destas eram provenientes de outras cidades do interior de Alagoas (Arapiraca, Rio Largo, Satuba, Cajueiro, Joaquim Gomes e Viçosa) enquanto que $5 \%$ procediam de outros estados do país (Interior de estado de São Paulo, Parnaíba- PE). Este dado reflete a necessidade de busca de melhor atendimento médico na capital, já que conforme relatos, há uma significativa carência de serviços especializados no interior do estado, evidenciando uma escassez de profissionais e de leitos tendo como consequência direta a sobrecarga do sistema assistencial.

A falta de vínculo entre os locais que realizam a assistência pré-natal destas gestantes e a maternidade onde será realizado o parto é um problema bastante relevante, pois resulta em um movimento de peregrinação destas mulheres já em trabalho de parto na busca por uma vaga de internação, o que pode acarretar em riscos adicionais à saúde da mãe e do recém-nato ${ }^{10}$. Estudos realizados em âmbito nacional êm evidenciando que este processo de peregrinação é uma realidade na região ordeste, onde mais de $30 \%$ das gestantes passam por tal dificuldade durante o ríodo de pré-natal ${ }^{11}$. Isto, associado a falhas na assistência como dificuldade de sso, início tardio e número menor de consultas de pré-natal do que o preconizado 


\section{ciênncia}

pelo Ministério da Saúde, acabam por afetar diretamente as condições de saúde das futuras mães e do feto.

Quando se parte para a análise dos hábitos de vida, 42,5\% das gestantes declararam etilismo, sendo que 22,5\% justificaram enquanto hábito social, dado que não pode ser quantificado no momento das entrevistas. Das entrevistadas 20\% informaram serem tabagistas. Quanto ao uso de drogas ilícitas, todas afirmaram não fazerem uso.

Em um contexto geral, pôde-se evidenciar um fato bastante preocupante, quando realizada análise pelo teste de Mann-Whitney para comparação de grupos, foi possível observar que as adolescentes que declararam fazer uso do álcool, apresentaram um tempo de gestação médio menor que o daquelas que não fizeram uso, média de 35,4 semanas, quando comparada com 39,1 semanas, respectivamente, resultando em um $\mathrm{p}=0.013$ (Tabela 1). Dessa forma, foi constatada uma tendência de as adolescentes etilistas evoluírem com gestações pré-termo (tempo de gestação < 37 semanas) e recém-nascidos prematuros, enquanto as não etilistas evoluíram em sua maioria com gestações a termo.

Considerando que durante a pesquisa fatores como tabagismo, presença de problemas de saúde durante a gestação e estado emocional da gestante tenham sido retratados na análise, estes valores não obtiveram relevância estatística, nesta análise, quando há diferença significativa entre os grupos comparados, o p-valor é < 0.05. Um resultado interessante, pois, demonstra índices contrários aqueles relatados na literatura analisada 1213 . 
Tabela 1: Fatores que potencialmente influenciam no tempo de gestação das adolescentes. Maceió-AL, 2020.

\begin{tabular}{|c|c|c|c|}
\hline \multicolumn{4}{|c|}{ TEMPO DE GESTAÇÃO } \\
\hline VARIÁVEL 1 & VARIÁVEL 2 & $\mathrm{n}$ & P-VALOR \\
\hline $\begin{array}{c}\text { Etilista } \\
(35,4 \pm 7,2 \text { semanas })\end{array}$ & $\begin{array}{c}\text { Não etilista } \\
(39,1 \pm 1,3 \text { semanas })\end{array}$ & 39 & 0.013 \\
\hline $\begin{array}{c}\text { Tabagista } \\
(37,4 \pm 4,1 \text { semanas })\end{array}$ & $\begin{array}{c}\text { Não tabagista } \\
(37,6 \pm 5,2 \text { semanas })\end{array}$ & 39 & 0.926 \\
\hline $\begin{array}{l}\text { Presença de problemas de saúde } \\
\qquad(37,3 \pm 3,9 \text { semanas })\end{array}$ & $\begin{array}{l}\text { Ausência de problemas de saúde } \\
\qquad(37,6 \pm 5,3 \text { semanas })\end{array}$ & 38 & 0.745 \\
\hline $\begin{array}{l}\text { Estado emocional tranquilo } \\
\quad(37,6 \pm 2,6 \text { semanas })\end{array}$ & $\begin{array}{l}\text { Estado emocional abalado } \\
\quad(37,4 \pm 7,3 \text { semanas })\end{array}$ & 38 & 0.104 \\
\hline
\end{tabular}

O consumo de bebidas alcoólicas durante a gestação pode trazer repercussões negativas tanto na saúde destas gestantes como do feto. Nas crianças, pode acarretar a Síndrome alcoólica fetal (SAF), o risco de ter uma criança portadora dessa síndrome é de aproximadamente $6 \%$ em mulheres alcoolistas ${ }^{14}$. A SAF, nas crianças, pode resultar em alterações do sistema nervoso central, anomalias neurológicas, craniofaciais, alterações no crescimento pré e pós-natal, malformações associadas e consequentemente, a um desfecho prematuro da gestação. Quanto as gestantes, o etilismo pode levar ao surgimento de alterações cardiovasculares, além de distúrbios neurológicos e psiquiátricos, podendo também, estar associada a um ganho ponderal insuficiente e menor número de consultas de pré-natal ${ }^{13}$.

No entanto, durante análise quantitativa das variáveis categóricas, através do teste de associação de variáveis, foi possível perceber que das 30 participantes que afirmaram não ser tabagistas, 66,7\% afirmaram não ser etilistas $(p=0,013)$, reforçando que um significativo número das jovens que participaram da pesquisa não possuía esses hábitos de vida (Tabela 2). Outro dado positivo desta avaliação, consiste no fato de que das 17 participantes que referiram etilismo, 94,1\% afirmaram que ainda assim tem boas perspectivas de futuro com relação a criança $(p=0,026)$ (Tabela 2$)$. O que eflete que apesar das alterações de saúde que o etilismo pode acarretar estas ainda antém perspectiva otimista do futuro afetivo com seu filho. 
Tabela 2: Análise qualitativa do índice de tabagismo, etilismo e gravidez desejada das gestantes adolescentes. Maceió-AL, 2020.

\begin{tabular}{|c|c|c|}
\hline VARIÁVEL 1 & VARIÁVEL 2 & P-VALOR \\
\hline Tabagismo & Etilismo & 0.013 \\
\hline Etilismo & Expectativa - futuro afetivo com o filho & 0.026 \\
\hline Gravidez Desejada & Continuou estudando após gravidez & 0.030 \\
\hline
\end{tabular}

Ao questionar as gestantes sobre sua percepção quanto a gestação, 57,5\% declararam que se tratava de uma gravidez indesejada, enquanto 42,5\% informaram querer a gestação desde o início. De acordo com as informações colhidas, pôde-se perceber por meio da narrativa das mulheres, que a gravidez na adolescência é considerada um tabu e quando associada a ausência de uma rede de apoio, agrava sua rejeição, refletindo em um isolamento e dificuldade de aceitação do seu futuro enquanto mãe.

Partindo-se da análise inferencial pela técnica de Mann-Whitney, observou-se que a idade média das participantes cuja gestação é desejada é estatisticamente maior do que a idade média das participantes cuja gestação não foi desejada (média de 17,5 anos, quando comparada com 16,4 anos, respectivamente, resultando em um p =0.041) (Tabela 3).

Tabela 3: Análise da relação entre idade e fatores relativos que podem interferir na gestação das adolescentes. Maceió-AL, 2020.

\begin{tabular}{|c|c|c|c|}
\hline \multicolumn{4}{|c|}{ IDADE } \\
\hline VARIÁVEL 1 & VARIÁVEL 2 & $\mathrm{n}$ & P-VALOR \\
\hline $\begin{array}{c}\text { Primíparas } \\
(16,8 \pm 1,8 \text { anos })\end{array}$ & $\begin{array}{c}\text { Multíparas } \\
(17,3 \pm 1,0 \text { anos })\end{array}$ & 40 & 0.584 \\
\hline $\begin{array}{l}\text { Gestação desejada } \\
(17,5 \pm 1,4 \text { anos })\end{array}$ & $\begin{array}{l}\text { Gestação não desejada } \\
(16,4 \pm 1,7 \text { anos })\end{array}$ & 40 & 0.041 \\
\hline $\begin{array}{l}\text { Presença de problemas de saúde } \\
\qquad(16,7 \pm 2,1 \text { anos })\end{array}$ & $\begin{array}{l}\text { Ausência de problemas de saúde } \\
\qquad(17,0 \pm 1,6 \text { anos })\end{array}$ & 39 & 0.793 \\
\hline $\begin{array}{l}\text { Estado emocional tranquilo } \\
\qquad(17,0 \pm 1,6 \text { anos })\end{array}$ & $\begin{array}{l}\text { Estado emocional abalado } \\
\qquad(16,9 \pm 1,8 \text { anos })\end{array}$ & 39 & 0.953 \\
\hline
\end{tabular}

Estes dados indicam que quanto mais jovem a gestante, menor o planejamento gestação e a aceitação do futuro enquanto mãe, pois a maternidade exige dessas lheres uma mudança da sua identidade, partindo-se da visão de que com a decisão levar a gestação a diante sua vida sempre estará vinculada as necessidades do filho. 


\section{ciência
piurala}

Dessa forma, muitos planos são deixados de lado ou redimensionados em função da gestação e da maternidade ${ }^{3}$.

\section{Processo da gestação}

Por meio desse trabalho, pode-se perceber que o tempo médio de gestação foi de 37,6 semanas com desvio padrão de 5 semanas para mais e para menos, destas 75\% das gestações ocorreram a termo e 22,5\% tiveram um desfecho prematuro (Gráfico 2). Evidenciando que a maioria das jovens mães tiveram parto a termo (dentro do período de normalidade: 37 semanas a 41 semanas e 6 dias). No que diz respeito à paridade, todas deixaram claro que nunca realizaram aborto anteriormente, destas $77.5 \%$ se declararam primíparas, 22,5\% afirmaram ser multíparas, dentre estas, todas declararam estar no seu segundo filho.

Gráfico 2: Perfil das gestantes participantes quanto ao momento do parto. MaceióAL, 2020.

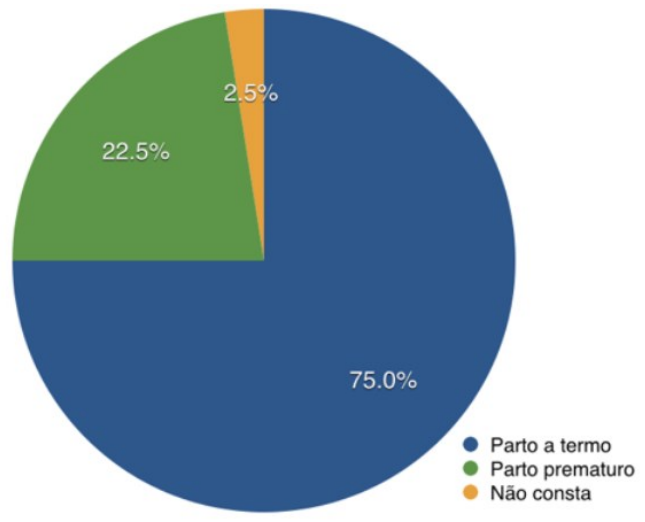

De acordo com Gariepy ${ }^{12}$, a gravidez não planejada/não intencional, que em sua maioria acomete mulheres jovens e de baixa renda, não possui associação confirmada com a prematuridade. Mas sabe-se com certeza que a prematuridade é a principal causa de morbimortalidade perinatal, principalmente em países desenvolvidos e que este nascimento precoce aumenta significativamente o risco de outros problemas como paralisia cerebral, deficiência visual, cognitiva ou auditiva e a sua principal forma de prevenção é identificar os fatores de riscos e minimizá-los durante o pré-natal.

Com relação à eventuais problemas de saúde/intercorrências, 80,0\% lararam não ter tido qualquer problema de saúde durante a gestação, enquanto que 
$17,5 \%$ relataram ter tido algum problema de saúde durante a gestação, dentre eles destacamos sangramento, que foi a principal causa de intercorrência, em segundo a infecção do trato urinário (ITU), que quando não tratada pode levar a diversas complicações, incluindo o aborto; 2,5\% não quiseram responder à pergunta. Outras complicações relatadas foram hipertensão gestacional e diabetes gestacional, hemorragia pós-parto e suspeita de aborto.

Um estudo realizado por Bouzas, Cader, Leão ${ }^{15}$ analisou o impacto da idade materna nas complicações clínicas, obstétricas e neonatais na primeira fase da adolescência, evidenciando que a hemorragia pós parto representou 33\% de incidência em mães jovens. Eles relataram que as complicações relacionadas ao aumento da pressão arterial nas gestantes jovens, estão em torno de 50\%, deixando claro seu alto índice. Em relação à Infecção do trato urinário (ITU), esta patologia foi analisada em conjunto com as vaginoses e, juntas, tiveram incidência de $29 \%$. Válido destacar que, neste mesmo estudo, apenas a diabetes gestacional não apresentou diferença significativa entre adolescentes precoces e tardias/adultas.

No tocante ao estado emocional das gestantes durante a gravidez, 57,5\% declararam que se sentiram tranquilas; 40,0\% tiveram seu estado emocional abalado (incluindo sentir-se assustada, ou com episódios de ansiedade, depressão, choque, medo e insegurança); 2,5\% não quis responder. Está situação foi relatada por algumas das participantes, por exemplo:

"Tive depressão no início da gestação porque minha vida havia mudado completamente, mas que nunca pensei em abortar." $N^{0} 05$

"Fiquei muito angustiada, triste e assustada sem querer conversar com ninguém no início." $N^{0} 19$

Estes resultados ressaltam que a gestação em mães adolescentes é vista como um período de muitas perdas, como da confiança da família, expectativas para o futuro devido o abandono da escola, abandono do companheiro por não aceitar a gestação e unto a isso, a gravidez é vivida como um período de ganho de responsabilidade que adolescente ainda não está preparada para assumir, muitas vezes gerando problemas icológicos como a baixa autoestima, vivência de alto nível de estresse, sintomas pressivos nos casos em que a gravidez foi indesejada ${ }^{16}$. 
Esse contexto se deve ao fato de que o processo da gestação entre mulheres jovens se torna ainda mais desgastante e conflitante, o que torna fundamental uma rede de apoio para que se possa minimizar as consequências da gravidez na adolescência. Confirmando que as gestantes que tiveram suporte familiar/parceiro e desejo de gestar, puderam se sentir muito mais tranquilas do que àquelas não tiveram base familiar estável.

\section{Quanto aos seus ciclos sociais}

Quando se parte para a análise das relações sociais $75 \%$ delas declararam manter algum tipo de relacionamento com o pai da criança, destas $55 \%$ afirmam que residiam com seu companheiro, mas apenas 10\% declararam estarem casadas com o parceiro, predominando o status de união estável ou namoro. As que afirmaram morar junto ao progenitor apresentaram respostas positivas com relação ao apoio durante a gestação, tanto financeiro quanto emocional. Ao serem perguntadas sobre a relação com o pai da criança, algumas participantes nos relataram:

"Sim. Moramos juntos, mas não somos casados, temos uma relação boa." $N^{o} 09$

"Sim. Moramos juntos, mas não somos casados, tive apoio do pai da criança durante toda a gestação. Nossas famílias foram muito presentes" $N^{o} 22$.

"Não. Ele não foi muito presente durante a gravidez e não sei se será agora que o menino nasceu." $N^{o} 40$

"Não. Não tive apoio durante a gravidez, nós não temos boa relação, pretendo criar meu filho sozinha com ajuda da minha família" $N^{o} 16$

Um estudo realizado na Argentina ${ }^{17}$ que compara a percepção dos jovens com relação a gravidez na adolescência, tem como conclusão uma maior dificuldade deste processo em mães solteiras, as quais eram ainda mais estigmatizadas e responsabilizadas pela gestação, sofrendo um julgamento moral por parte da sociedade. Este perfil se confirmou durante as nossas análises, quando foi possível perceber que o apoio do parceiro ameniza esse sentimento, trazendo maior segurança para com a gestação. 
Em relação ao apoio familiar, $80 \%$ das gestantes analisadas declararam ter apoio da família, sendo predominante a figura compreensiva das mães, 10\% contam que não obtiveram suporte familiar apenas no início da gestação, 5\% deixaram claro que não tiveram apoio durante todo o período da gravidez e durante o parto e $5 \%$ não quiseram responder ao questionamento. Pode-se observar com base nos dados coletados e na bibliografia apresentada, que o cenário de não aceitação no contexto familiar reflete diretamente nos outros ciclos de convívio social (parceiro, amigos e âmbito escolar). Pode-se exemplificar essa situação através dos relatos das participantes, ao serem questionadas sobre o apoio familiar:

"No início não, e foi muito estranho pois minha mãe sempre me disse para não engravidar. Mas agora, minha família inteira já aceitou e está me ajudando". $N^{0} 013$

"Inicialmente não, mas quando cheguei na metade da gestação eles começaram a me apoiar, pois viram que o pai da criança não era presente e que eu não poderia passar por isso sozinha. $N^{0} 39^{\prime \prime}$

Segundo Cavalcanti et $\mathrm{al}^{18}$, o impacto do meio em que a gestante está inserida, com suas situações de vulnerabilidade, afetam a saúde psicológica, agravando o sofrimento e influenciando-as nos sentidos e significados atribuídos à gravidez, podendo interferir na adaptação a ela.

No que diz respeito às interações sociais 65\% declararam que não houve mudança na sua relação com os amigos, 35\% delas declararam que sentiram afastamento dos amigos e que não tiveram apoio deles durante o processo da gestação. Dentre as puérperas e gestantes que declararam afastamento, a justificativa mais frequente estava na ausência de outras amigas que também fossem mães, e que, portanto, o ciclo de amigos não compreende o contexto vigente de vida da adolescente enquanto mãe, ocasionando um afastamento e solidão destas jovens. O que revela que o processo de gestação na adolescência não é natural desta etapa da vida, gerando uma ensação de deslocamento e abandono do meio social. Isto foi comentado pela articipante $\mathrm{N}^{\mathrm{o}} 34$ :

"Todas as minhas amigas se afastaram, as minhas únicas amigas são minha mãe e minha sogra." 


\section{ciênciála
pural}

Quanto ao processo educacional, ficou demonstrado na pesquisa que $85 \%$ das entrevistadas declararam que não deram continuidade aos estudos, mas que pretendem retornar após a gestação, e as principais justificativas que surgiram nas narrativas destas mulheres consistiam em: dificuldade de acompanhar os estudos durante a gravidez, a pandemia do coronavírus com a paralisação das atividades escolares, e a pretensão de começar a trabalhar para auxiliar com as despesas de casa. Este número é bastante expressivo evidenciando uma taxa elevada de evasão escolar durante o período da gestação. De acordo com as entrevistas 77,5\% pretendem continuar ou voltar a estudar após o período de licença da gestação, das outras 22,5\%, $10 \%$ declararam que pretendem trabalhar, $10 \%$ não declararam interesse em trabalhar ou estudar e 2,5\% das adolescentes já trabalhavam.

Pesquisas que analisam mudanças na vida social das jovens gestantes referem a evasão escolar como a mudança mais frequente. Uma informação preocupante quando se parte do princípio de que o abandono escolar resulta em uma menor capacitação destas mulheres para se inserir no mercado de trabalho futuramente, acarretando obstáculos para seus projetos de vida. No entanto, é necessário compreender que a gravidez na adolescência não consiste em um processo homogêneo, e que é altamente dependente do contexto social no qual essas jovens estão inseridas. Quando ocorre em classes sociais mais baixas, as adolescentes apresentam maior dificuldade de concluir seus estudos, o que na maioria das vezes pode ser consequência de uma falta de apoio familiar, do parceiro e dos outros ciclos sociais em sua vida 6 .

E quando se parte da análise dos fatores de prevenção a gravidez na adolescência, a educação surge como uma das principais medidas, sendo o ambiente escolar o local ideal para propagação de ações que promovam uma educação sexual integrada a formação dessas jovens, além da introdução de programas de planejamento familiar em mulheres desta faixa etária. É através de medidas como stas, que as adolescentes aprenderão a realizar práticas sexuais de maneira esponsável, prevenindo uma gravidez inoportuna, infecções sexualmente nsmissíveis, além de como se defender diante de episódios de abuso e violência ual 19. Essa perspectiva pode ser confirmada através dos dados de análise 
qualitativa, no qual as gestantes que obtiveram um planejamento prévio da gravidez, evoluíram com as menores taxas de evasão escolar (Tabela 2).

\section{Percepção da gravidez}

De acordo com a análise realizada durante a realização do trabalho de campo, pôde-se perceber que as futuras mães têm uma relação positiva $(77,5 \%)$ quanto as expectativas para o futuro de seus descendentes, por outro lado, 22,5\% declararam ter expectativas negativas/inseguranças, ainda assim as participantes apresentaram narrativa dúbia sobre a maternidade como por exemplo:

"Estou nervosa, tenho medo de não saber cuidar do meu filho" $n^{\circ} 37$

"Estou nervosa, tenho medo de não saber como ser mãe" $n^{\circ} 39$

O estudo realizado por Sámano et al ${ }^{20}$ argumenta que mesmo diante de expectativas centradas nos filhos por muitas mães adolescentes, as perspectivas e os valores do núcleo familiar refletem sobre o que é esperado no padrão social para a gravidez e a parentalidade, e que nem sempre esses valores refletem com precisão os valores dos adolescentes em relação à gravidez.

\section{Conclusões}

Diante da análise dos dados coletados e do embasamento na bibliografia encontrada, nota-se que a gravidez na adolescência não só é uma realidade no município de Maceió, como permanece um importante problema de saúde pública gerando impactos negativos nos aspectos sociais, econômicos e nos indicadores de saúde das jovens gestantes. Apesar desta realidade, a temática abordada ainda é muito negligenciada, refletindo na escassez de estudos atualizados quanto ao assunto debatido, especialmente no Brasil.

$\mathrm{Na}$ análise estatística pode-se destacar a relação entre o etilismo e o tempo de estação < 37 semanas, gravidez pré-termo, um dado que evidencia como os hábitos vida das jovens mães pode interferir na gravidez; observou-se ainda que a média idade das participantes cuja gestação é desejada é estatisticamente maior do que a 
idade média daquelas em que a gestação não foi desejada, dado que confirma que mulheres mais jovens tem um menor planejamento da sua saúde reprodutiva.

Além disso, na interpretação dos dados qualitativos: as gestantes que possuíam uma gravidez desejada tiveram menor taxa de evasão escolar quando comparada as gestações não desejadas; as jovens que não se declaravam etilistas, em sua maioria, também não eram tabagistas, um dado bastante positivo quando se analisa a consequência destes hábitos de vida na saúde da mãe e da criança; as adolescentes que se declararam etilistas, apesar deste hábito de vida, afirmam ter expectativas positivas para sua relação com o filho.

Através do contexto abordado, não há dúvida da necessidade de pesquisas como está no sentido de produzir uma bibliografia mais atual e condizente com a realidade do nosso país quanto a gestação precoce para que, a partir disso, possam ser desenvolvidas medidas preventivas voltadas a sexualidade e as práticas sexuais na adolescência, como também medidas de apoio as adolescentes grávidas para que todo o processo se torne menos doloroso, na tentativa de minimizar os impactos negativos que a maternidade precoce pode causar a essas jovens.

\section{Referências}

1.Taborda JA, Silva FC da, Ulbricht L, Neves EB. Consequências da gravidez na adolescência para as meninas considerando-se as diferenças socioeconômicas entre elas. Cad Saúde Coletiva 2014;22:16-24. https://doi.org/10.1590/1414$462 \times 201400010004$.

2.Cavenaghi SM. População, espaço e sensibilidade, 2010, p. 231-74.

3.Dias ACG, Teixeira MAP. Gravidez na adolescência: um olhar sobre um fenômeno complexo. Paid (Ribeirão Preto) 2010;20:123-31. https://doi.org/10.1590/S0103863X2010000100015.

Wado YD, Sully EA, Mumah JN. Pregnancy and early motherhood among dolescents in five East African countries: A multi-level analysis of risk and protective actors. BMC Pregnancy Childbirth 2019;19:1-11. https://doi.org/10.1186/s1288419-2204-z.

edler Milla Wildemberg, Araújo Alisson, Souza Márcia Christina Caetano de. The vention of teenage pregnancy in adolescent's view. Texto contexto - enferm. 
[Internet]. 2015 Mar [cited 2020 Dec 05] ; 24( 1 ): 30-37. Available from: http:/ / www.scielo.br/scielo.php?script=sci_arttext\&pid=S010407072015000100030\& lng=en. https://doi.org/10.1590/0104-07072015000130014.

6.Vicentim AL, Queiroz AMDA, Sasaki NSGM dos S, Santos M de LSG. Prevenção da gravidez na adolescência no Brasil. Enferm Bras 2019;18:582. https:/ / doi.org/10.33233/eb.v18i4.2497.

7.Organização Mundial da Saúde. Ficha informativa Gravidez na Adolescência 2018:1. http:// www.who.int/mediacentre/factsheets/fs364/en/,2018. (acessado 30 de novembro de 2020).

8.Departamento de Informática do Sistema Único de Saúde. Sistema de Informação $\begin{array}{llll}\text { sobre Nascidos Vivos } & \text { (SINASC) }\end{array}$ http://tabnet.datasus.gov.br/cgi/tabcgi.exe?sinasc/cnv/nval.def (acessado 1 de dezembro de 2020).

9.Brasil. Ministério da Saúde. Resolução CNS/MS no 466, de 12 de dezembro de 2012. [s.d.].

http://bvsms.saude.gov.br/bvs/saudelegis/cns/2013/res0466_12_12_2012.html (acessado 28 de novembro de 2020).

10.Leal M do C. Prenatal care in Brazil. Cad Saude Publica 2014;30:S1-15.

11.Leal M do C, Esteves-Pereira AP, Viellas EF, Domingues RMSM, Gama SGN da. Prenatal care in the Brazilian public health services. Rev Saude Publica 2020;54:08. https:/ / doi.org/10.11606/s1518-8787.2020054001458.

12.Gariepy AM, Lundsberg LS, Stolar M, Stanwood NL, Yonkers KA. Are pregnancy planning and timing associated with preterm or small for gestational age births? Fertil Steril 2015; 104:1484-92. https:/ / doi.org/10.1016/j.fertnstert.2015.08.012.

13.Freire K, Padilha P de C, Saunders C. Fatores associados ao uso de álcool e cigarro na gestação. Rev Bras Ginecol e Obstet 2009;31:335-41. https://doi.org/10.1590/S0100-72032009000700003.

14.Pinheiro SN, Laprega MR, Furtado EF. Psychiatric morbidity and alcohol use by pregnant women in a public obstetric service | Morbidade psiquiátrica e uso de álcool em gestantes usuárias do Sistema Único de Saúde. Rev Saude Publica 2005;39.

5.Bouzas ICS, Cader SA, Leao L. Gravidez na adolescência: uma revisão sistemática impacto da idade materna nas complicações clínicas, obstétricas e neonatais na primeira fase da adolescência. Adolesc Saude. 2014;11(3):7-21

6.Carvalho, CC. Gravidez na Adolescência - Principais causas e taxas; 2011 [cited 2020 Nov 28]. Available from: https://repositorio.ufmg.br/bitstream/1843/BUOSAAWN49/1/clara_coelho.pdf 
17.Weisbrot MA, Fraguas L, Espósito ME, Camoirano J, Sciarreta S, Granero M, et al. Perceptions of adolescents from Bajo Boulogne, Buenos Aires, Argentina, on teenage pregnancy: A qualitative study. Arch Argent Pediatr 2019;117:314-21. https://doi.org/10.5546/aap.2019.eng.314.

18.Cavalcanti A, Maia E, Rêgo MH. Resiliência e Gravidez na Adolescência: Uma Revisão Integrativa. Psicol Saúde Doenças 2018;19:710-23.

19.UNFPA. Operational Guidance for Comprehensive Sexuality Education: A Focus on Human Rights and Gender 2014:1-76.

20.Sámano R, Martínez-Rojano H, Robichaux D, Rodríguez-Ventura AL, SánchezJiménez B, de la Luz Hoyuela M, et al. Family context and individual situation of teens before, during and after pregnancy in Mexico City. BMC Pregnancy Childbirth 2017;17:1-16. https:/ / doi.org/10.1186/s12884-017-1570-7. 